\title{
State of the art in intelligent adaptive energy- saving pulse power technology of WEDM
}

\author{
Yinsheng Fan ${ }^{1, a, *}$, Jicheng Bai ${ }^{1, b, *}$, Bo Zhang ${ }^{1, c}$, and Zhiyu Yang ${ }^{1, d}$ \\ ${ }^{1}$ School of Mechatronics Engineering, Harbin Institute of Technology, Harbin, Heilongjiang, \\ People's Republic of China \\ a fanyinshenghero@163.com, ${ }^{\mathrm{b}}$ jichengbai@hit.edu.cn, ${ }^{\mathrm{c}} 996311109 @$ qq.com, \\ d946392445@qq.com
}

Keywords: WEDM, intelligent adaptive, energy-saving pulse power, energy utilization rate, automation

Abstract: Pulse power as the heart of wire electrical discharge machining (WEDM) tool determines machining quality, machining accuracy, processing efficiency, wire electrode wear, stability of machining process and energy utilization rate of WEDM. For low energy utilization rate and low automation degree of pulse power, this paper systematically analyzed the development of energy-saving technology and intelligent adaptive control technology of pulse power and pointed out its key development direction.

\section{Introduction}

Green and intelligent manufacturing is one of the main development directions of manufacturing industry and it is also an important pillar of strategic emerging industries. Manufacturing should be committed to energy conservation, improving added value and competitiveness of product [1-2].

Pulse power as the heart of Wire electrical discharge machining (WEDM) tool determines machining quality, machining accuracy, processing efficiency, wire electrode wear, stability of machining process and energy utilization rate of WEDM [3]. Therefore, pulse power technology has been a research hot spot in the field of WEDM. With the shortage of energy and development of automation technology, green and intelligent manufacturing is the development trend of manufacturing industry. For WEDM, improving the processing efficiency, increasing automation level and reducing energy consumption are the main problems currently. And the key to solving aforementioned problems is the pulse power. Reciprocating travelling wire electrical discharge machining tool has the advantages of simple structure, ability to process large thickness of workpiece and low using cost [4]. Due to current research on intelligent adaptive energy-saving technology of pulse power being immature, it has not been well configured on the machine tool. So low energy utilization rate, low machining quality, low processing efficiency and other problems exist in WEDM tool. Intelligent adaptive energy-saving pulse power can greatly improve energy utilization rate, automation level, processing efficiency, machining quality of machine tool and can automatically select optimal processing parameters through removing energy dissipation element 
resistor and adopting intelligent adaptive control technology. So the intelligent adaptive energysaving pulse power technology of WEDM has become more and more important.

Therefore, research on intelligent adaptive energy-saving pulse power technology has important economic benefit to improve energy utilization rate of WEDM. Also it has important practical significance to improve automation level and competitiveness of WEDM tool. This paper systematically analyzed the development of energy-saving technology and intelligent adaptive control technology of pulse power.

\section{State of the art in energy-saving pulse power technology of EDM}

With the electrical discharge machining (EDM) developing towards green and energy-saving, it puts forward higher requirements for energy utilization rate of pulse power and also promotes the development of energy-saving pulse power technology. Companies and research institutes have developed different features of energy-saving pulse power.

Korea Electro-technology Research Institute and Daewoo Heavy Industries \& Machinery Ltd. developed a full bridge inverter type energy-saving pulse power of WEDM [5], as shown in Figure 1. Full-bridge topology is composed of four IGBT (Insulated Gate Bipolar Translator). Driving signals of IGBT Q1 and IGBT Q4 are consistent. Driving signals of IGBT Q2 and IGBT Q3 are consistent. Two groups relative bridge arm of IGBT conduct alternately. So the dc power supply is converted to high frequency pulse signal through high frequency transformer. The highest voltage applied in power switch under steady state is the input voltage $\mathrm{Vg}$. The voltage of power switch under transient state is also clamped at power supply voltage Vg. Excessive energy of gap discharge is transferred back to the power supply through diode and it improves energy utilization rate. But this pulse power requires four driving circuits which are not common ground with each other. The pulse power is composed of many components, which increases the complexity of the pulse power.

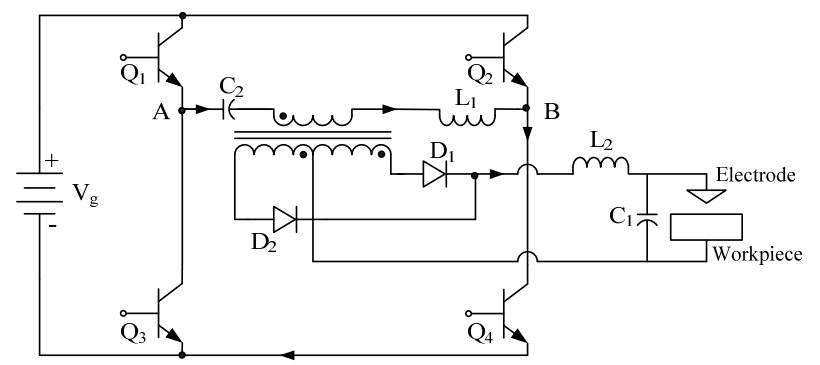

Figure 1 Full bridge inverter type energy-saving pulse power of WEDM [5]

Mitsubishi Denki Kabushiki Kaisha [6] applied for a patent of full bridge type energy-saving pulse power and its schematic diagram is shown in Figure 2. Power switches Q1 Q4 constitute four bridge arms of full bridge. Voltage detecting circuit and average voltage detecting circuit collect discharge gap voltage. The collected voltage pulse and average voltage are sent to voltage comparison circuit and average voltage comparison circuit. Control system controls on and off of power switches to generate different gap voltage waveforms according to results of the comparison circuit. Different voltage waveforms are generated by controlling turn-on, turn off time and sequence of power switches. The main circuit of pulse power does not have current-limiting resistor and its energy utilization rate is improved. This pulse power requires four driving circuits. Deadtime needs to be left between upper and lower bridge arm drive signals because power switch needs time to turn off. So the driving circuit is complicated.

Japanese researchers Masao Murai et al proposed capacitor discharge type energy-saving pulse power of EDM [7]. Its circuit diagram is shown in Figure 3. When SW1 is on, capacitor C is 
charged by power supply E through SW1 and inductor L. Current of inductor L feeds back to power supply E through diode D2 when voltage across capacitor $\mathrm{C}$ reaches voltage of power supply $\mathrm{E}$. Therefore, voltage across capacitor $\mathrm{C}$ could be charged to voltage of power supply $\mathrm{E}$ for each charge process. This ensures that gap discharge energy from capacitor is consistent during each pulse discharge. That is to say, it is equal energy processing. Also it can ensure processing surface quality. There is no energy dissipation element in main circuit of pulse power. So energy utilization rate of the pulse power is high.

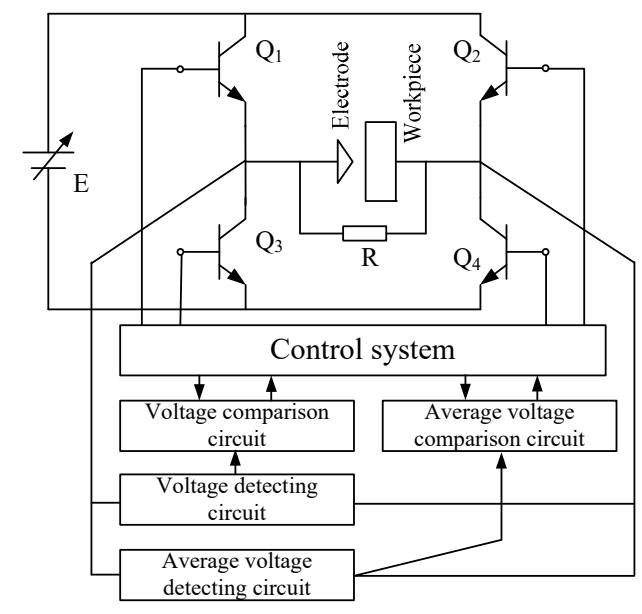

Figure 2 Mitsubishi Electric's full bridge type energy-saving pulse power of WEDM [6]

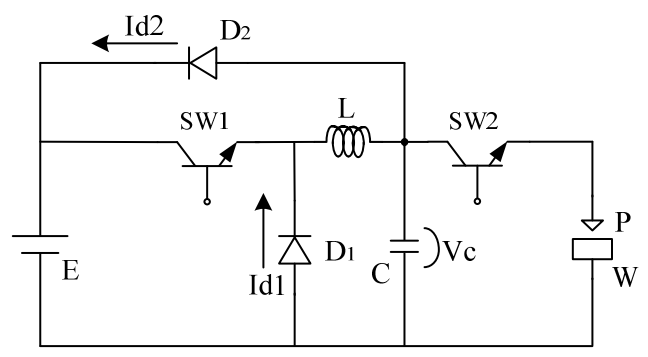

Figure 3 Capacitor discharge type energy-saving pulse power of EDM [7]

Figure 4 is the principle diagram of energy-saving pulse power of EDM developed by Industrial Technology Research Institute of Hsin-Chu [8]. The pulse power adopts high-voltage to breakdown discharge gap. After success of causing the ignition, the high- voltage output is stopped. Lowvoltage is used in spark discharge machining. Discharge circuit uses energy storage element inductor instead of energy dissipation element resistor. In addition, the pulse power has two processing modes, that is, equal frequency mode and equal energy mode. Energy utilization rate of this pulse power is improved.

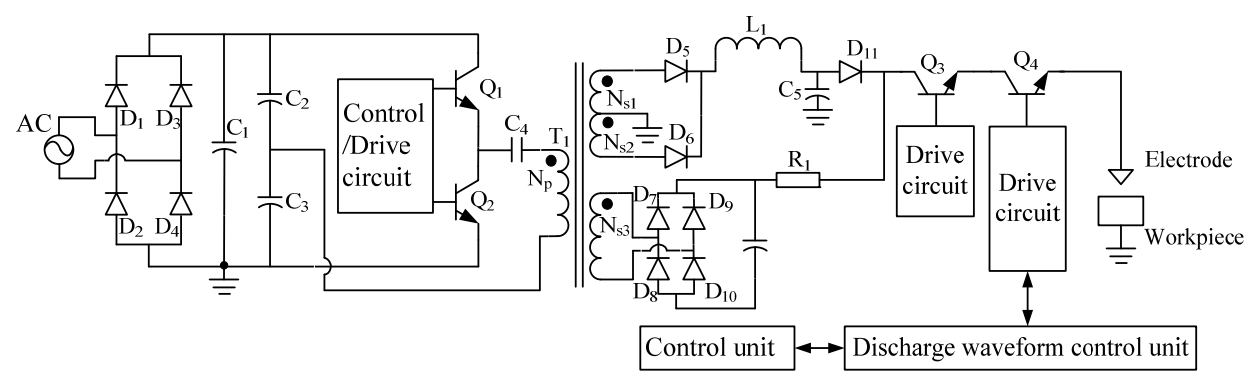

Figure 4 Energy-saving pulse power of EDM [8] 
Nanjing University of Aeronautics and Astronautics proposed a kind of pulse power that having high and low voltage composite circuit [9], as shown in Figure 5. Because power switch M5 is on during pulse interval, inductor current could be set up in advance. After success of causing the ignition, inductor current is forced to flow to discharge gap to machine by turning off power switch M5. Machining current waveform of the pulse power has no climbing phenomenon. So processing efficiency can be improved and the pulse power can output smaller pulse duration. In addition, the size of inductor current can be altered by adjusting duty cycle of preceding stage double transistor forward converter. Pulse duration and pulse interval of machining gap can be altered by adjusting pulse duration and pulse interval of power switch M5.

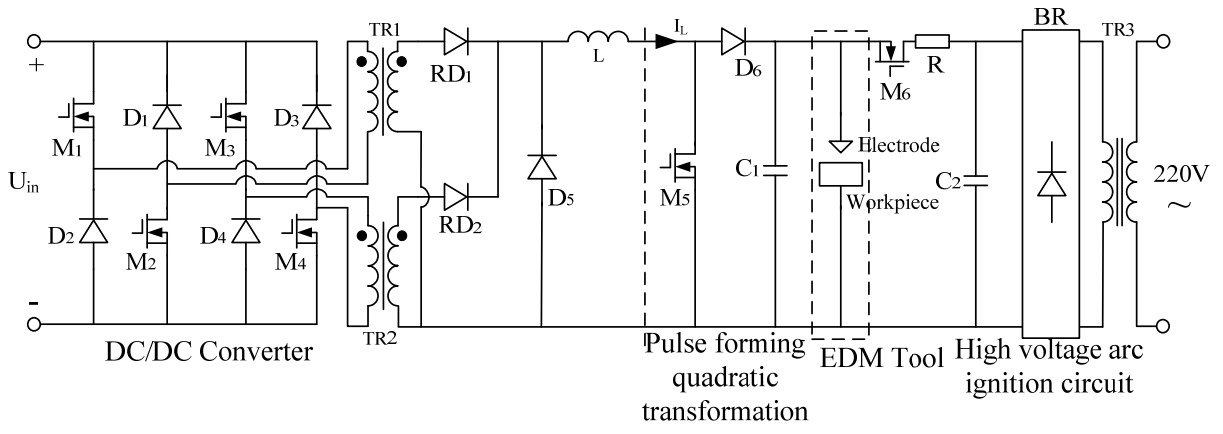

Figure 5 Composite structure current mode pulse power of EDM [9]

$\mathrm{Ph}$. D. Song in Harbin Institute of Technology designed a kind of energy-saving pulse power of EDM [10]. Figure 6 is the schematic diagram of main circuit of PWM (pulse width modulation) control energy-saving pulse power of EDM. This pulse power has high and low voltage composite main circuit. It uses high voltage to ignite and low voltage to process. Pulse parameters are realized by PWM control mechanism. This pulse power also uses energy storage element inductor instead of energy dissipation element resistor. Its energy-saving effect is superior to traditional currentlimiting resistor pulse power.

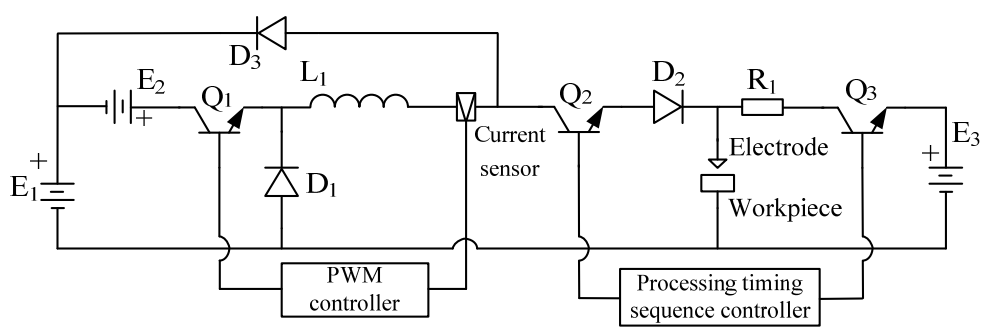

Figure 6 Schematic diagram of main circuit of PWM control energy-saving pulse power of EDM

[10]

$\mathrm{Ph}$. D. Li in Harbin Institute of Technology developed a multi-mode energy-saving pulse power with three power half-bridge topology [11]. The pulse power has equal energy and equal period mode. Also it has high and medium voltage, low and medium voltage composite mode, which are suitable for rough and finish machining. The pulse power adopts method of combination energysaving topology and discharging energy-saving to improve machining accuracy and energy utilization rate [12].

Figure 7 is chopper type energy-saving pulse power of EDM developed by Xi'an University of Architecture and Technology [13]. The adjustment of voltage is realized by voltage regulating circuit. The regulation of pulse duration of the first power of voltage is realized by voltage regulating circuit. The regulation of pulse duration of the first power switch (Q1) is realized by master microcontroller, pulse transformation and arcing drive circuit. The regulation of pulse 
duration of the second power switch (Q2) is realized by master microcontroller, pulse transformation and chopper drive circuit. The adjustment of current is realized by stable current circuit. Power switch Q1 and Q2 turn on simultaneously. The first power switch does not participate in current supply of main pulse, so it has little turn-on loss and turn-off loss and has no conduction loss. Pulse duration of power switch Q1 and Q2 depend on PWM pulse signal. Q2 only plays the role of switch, which significantly reduces working voltage of power switch Q2. Current-limiting resistor is eliminated, and energy utilization rate of the pulse power is improved. The pulse power has performance of easy ignition, adaptive voltage regulation and pulse parameters adjustable.

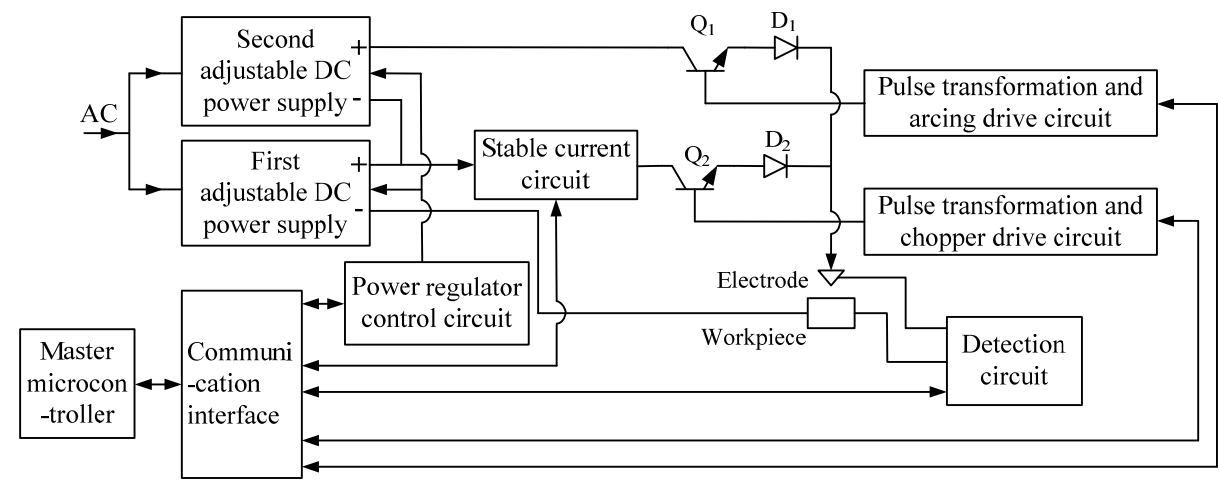

Figure 7 Principle diagram of chopper type energy-saving pulse power of EDM [13]

Based on above analysis, various kinds of energy-saving pulse power of EDM have sprung up in recent years. Among them, vast majority of main circuit of pulse power adopt inductor component or inductor in transformer winding instead of resistor to limit current and to store energy. Thus, energy utilization rate of pulse power is greatly improved. Very few main circuit of pulse power have no resistor and inductor components, and adopt method of chopping current of each power switch being cyclically superimposed to limit current. The power switch adopts full-bridge or halfbridge topology to generate different pulse voltage waveforms. Energy fast feedback circuit is designed and energy stored in inductor could be released to power supply and processing circuit to recycle, which improves energy utilization rate of pulse power. And high and low voltage composite circuit is used to eliminate the phenomenon of current climbing and smearing.

In order to further improve the energy utilization rate of pulse power, energy- saving pulse power of WEDM should focus on the following aspects. (1) The form and distribution of energy storage element in main circuit of energy-saving pulse power. Energy storage element adopts inductor or capacitor. Influence of distribution of inductor or capacitor on energy transfer in main circuit needs to be studied. (2) Optimization of cyclically superimposed methods of power switch. What kind of cyclically superimposed methods of power switch is adopted to adjust current in the circuit and to minimize loss of power switch. (3) Design of new energy fast feedback circuit can improve energy utilization rate of pulse power. The energy stored in inductor or capacitor can be released into power supply and processing circuit to cycle use through energy fast feedback circuit. (4) Zero switching loss technology of power switch. Experiment proved that the ratio of loss of power switch accounted for total output energy of main circuit of energy-saving pulse power is large[17]. So how to reduce the loss of power switch is the key to improve energy utilization rate of energy-saving pulse power. (5) How to improve gap spark discharge rate and reduce short circuit rate? Energy utilization rate of energy-saving pulse power can be improved through reducing energy waste due to short circuit. (6) Reducing consumption of energy transfer about high frequency wire in main circuit of pulse power. 


\section{State of the art in intelligent adaptive control technology of pulse power}

Another development tendency of pulse power of EDM is intelligent. The difference between intelligent adaptive pulse power and traditional pulse power is very big. For traditional pulse power, mostly operating workers choose power parameters according to machining process, workpiece material and so on. Power parameters are not adaptive to change along with gap state. So processing effect is restricted significantly. However, intelligent adaptive pulse power incorporates adaptive control technology and it could automatically adjust machining parameters according to machining conditions. It can ensure processing state to be best stable. And it greatly improves automation of machine tool and quality of products. So it has important practical significance to develop intelligent adaptive pulse power. At present, control methods and strategies widely used in intelligent adaptive pulse power mainly include fuzzy control, neural network, expert system, robust control, etc.

In terms of intelligent pulse power, Sodick company of Japan took the lead in applying artificial neural network technology to pulse power of EDM and developed NF numerical control pulse power [14]. This pulse power could automatically calculate optimum machining parameters based on neural network algorithm and process database, and it does not need to set machining parameters manually. Fuzzy control could also be chose to optimal control in the processing.

Wang in Norwegian University of Science and Technology adopted a hybrid artificial neural network and genetic algorism methodology to build optimization model for EDM process [15]. Pulse duration, pulse interval, peak current, open circuit voltage, gap distance and servo sensitivity are input parameters of model. Output parameters of model are material removal rate and surface roughness. It provides some reference for EDM parameters optimization methods.

Kao C C and Shih A $\mathrm{J}$ in University of Michigan developed a micro-hole EDM system with adaptive fuzzy logic controller and precision piezoelectric stage [16]. Input parameters of fuzzy logic controller are average gap voltage, deviation in spark ratio, and change in the deviation in spark ratio. The fuzzy logic controller generates two output parameters, servo command speed and servo command displacement. It identifies gap spark state, arcing state and short-circuit state to control speed and displacement of piezoelectric stage. It suppresses occurrence of arcing and shortcircuit. Experiments show that the fuzzy logic control system of EDM can effectively regulate discharge gap and yields a much more stable and efficient micro-hole EDM drilling process.

$\mathrm{Ph}$. D. Wang in Harbin Institute of Technology developed a microcomputer adaptive control pulse power [17]. Linear mathematical model about servo reference voltage, pulse interval and each controlled variable is built using adaptive control algorithm. Model parameters are calculated by identification algorithm. Adaptive control of EDM is realized by online adjusting servo reference voltage and pulse interval to optimum.

$\mathrm{Ph}$. D. Luo in Harbin Institute of Technology decomposed fuzzy relation equation according to decoupling characteristic of fuzzy controller [18]. So the decoupling of servo reference voltage, electrode jump motion cycle and pulse interval is realized. And the design of multi-input and multioutput fuzzy controller is simplified. Combination of artificial neural network and fuzzy logic theory not only can make up for lack of knowledge acquisition and expression about neural network, but also can play the advantages of self-learning function and low fuzzy entropy about neural network, which corrects negative impact of imperfect control rules on control effect.

Taiwan scholar M. T. Yan designed a multivariable and three-region (safe, critical, and dangerous) fuzzy controller by constructing fuzzy rules based on operator experience and expert knowledge [19]. Input parameters of fuzzy controller are abnormal sparks and sparking frequency, and its output parameters are servo feed and pulse interval. It solved adaptive control optimization problem for WEDM. In addition, M. T. Yan developed pulse identification and control system for 
micro WEDM [20]. The gap state of micro EDM was divided into four kinds, open circuit, spark, arc and short circuit. Pulse waveforms during machining are collected to realize the real time identification of gap state and formulate the corresponding pulse control strategy. So pulse interval is online adjusted according to detected discharge state information, which reduces occurrence of arcing and short circuit. Experiments show that the control system has strong robustness and can significantly eliminate the adverse effects of accidental interference on machining process.

Jingyu Pei in Shanghai Jiao Tong University obtained qualitative relationship between various control parameters and machining targets through experiments [21]. A multi-input, multi-output and multi-level hierarchical intelligent control system of micro EDM was designed, as shown in Figure 8. Fuzzy logic theory was applied to decision-making layer and execution layer of intelligent system. This control system can automatically seek optimal processing control strategy on the premise of guaranteeing the machining accuracy and it significantly shortens the machining time.

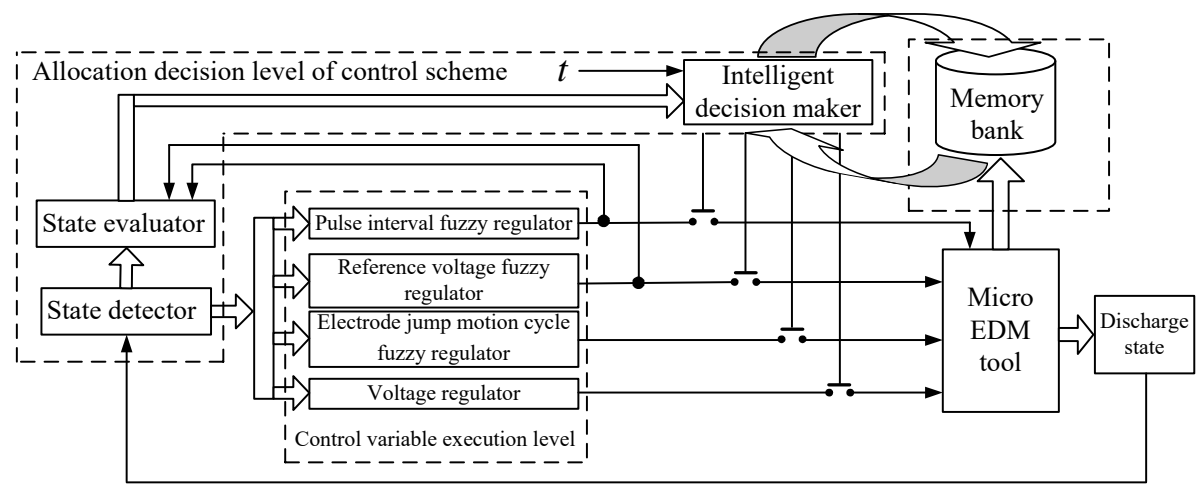

Figure 8 Structure diagram of intelligent control system of micro EDM [21]

Professor Liu in China University of Petroleum designed a fuzzy RBF (radial basis function) neural network controller of EDM [22]. The controller takes no-load rate, normal discharge rate, abnormal discharge rate (including arcing and short circuit), normal discharge rate with regard to pulse period and electrode jump motion cycle as inputs. And its output parameters are servo reference voltage, pulse interval and electrode jump motion cycle. The controller is modeled and simulated by MATLAB.

Huang in Harbin Institute of Technology Shenzhen Graduate School developed a pulse power for micro EDM, which adopted method of effective discharge spark number detection to carry out statistics on discharge state [23]. Fuzzy control algorithm is used to adjust pulse duration and pulse interval to improve the processing performance. The fuzzy control algorithm not only can judge current discharge state, but also can predict change trend of current discharge state. But the control strategy of adjusting pulse interval would affect machining accuracy and machining quality.

Zhou in Tsinghua University took the sum of spark ratio and transient arc ratio as gap state, which numerically denotes the variations of EDM process [24]. Common descriptive processing model was established based on filtering of spectral analysis of discharge state. So a linear timevaried predictive model for discharge state was built based on abovementioned model structure. Experimental verifications showed that this predictive model could quickly and accurately provide one step ahead predictions with mean error less than $2 \%$.

Yang in East China Jiaotong University proposed a method of controlling discharge gap and pulse interval based on fuzzy control [25]. The deviation between mean value of arc ignition delay time and reference value of arc ignition delay time, and variation rate of the deviation are inputs of discharge gap fuzzy controller. Its output is feed frequency of driver. Pulse interval fuzzy controller takes deviation of arc discharge times and deviation of arc ignition delay time as inputs. Its output is 
pulse interval. The developed fuzzy controller is helpful to improve processing efficiency and processing surface quality. But the setting of parameters in fuzzy controller has yet to be improved.

Based on comprehensive analysis of current intelligent adaptive control technology of pulse power for EDM, its intelligent adaptive control is mainly reflected in the following aspects. (1) EDM process optimization model is established by using fuzzy control, neural network and some modern control algorithm to optimize machining parameters automatically. (2) Research on real time identification and prediction of gap state. (3) Adaptive control of discharge gap and pulse interval. In addition to above several aspects need to be further development about intelligent adaptive control technology of pulse power for EDM, adaptive control on machining of variable thickness workpiece needs to be studied.

\section{Conclusions}

This paper comprehensively analyzed the development of energy-saving technology and intelligent adaptive control technology of EDM pulse power. In order to further improve energy utilization rate of pulse power and its energy saving effect, energy-saving pulse power of WEDM should focus on the following aspects. (1) The form and distribution of energy storage element in main circuit of energy-saving pulse power. (2) Optimization of cyclically superimposed methods of power switch. (3) Design of new energy fast feedback circuit. (4) Zero switching loss technology of power switch. (5) How to improve gap spark discharge rate and reduce short circuit rate? (6) How to reduce consumption of energy transfer about high frequency wire in main circuit of pulse power.

In the aspect of intelligent adaptive control technology, fuzzy control, neural network, expert system, robust control and other control methods and strategies are used to adjust and optimize EDM process. The new development of intelligent adaptive control of pulse power is mainly reflected in the following aspects. (1) Establishment of EDM process optimization model. (2) Real time identification and prediction of gap state. (3) Adaptive control of discharge gap and pulse interval. (4) Adaptive control on machining of variable thickness workpiece.

\section{Acknowledgements}

This research is supported by National Natural Science Foundation of China (Grant NO. 51575137, 51175120, 51305230), and Harbin Applied Technology Research and Development Project (2015RAXXJ027).

\section{References}

[1] Yang SZ, Wu B, Li B (2006) Further discussion on trend in the development of advanced manufacturing technology. J Mech Eng 42:1-5.

[2] Lu YX (2010) Green, intelligent manufacturing and strategic emerging industries. In: The Chinese mechanical engineering society annual meeting in 2010, Luoyang, China, 1-15.

[3] Bai JC, Liu JC, Guo YF, Yang XD (2014) Non-traditional machining. China Machine Press, Beijing.

[4] Yuan FG (2011) Contents and trends of non-traditional machining method. Mech \& electr Eng Technol 40:142-143.

[5] Jeong IW, Kim JS, Pavlovets MV et al (2003) Design of 10kW switching power supply and discharge circuit for wire-cut electric discharge machine. In: 14th IEEE International Pulsed Power Conference. Dallas, USA,13291331.

[6] Yoshikazu U, Seiji S (2004) Power supply for wire electric discharge machining. US 6756557B2.

[7] Yamanashi FL (2006) Power supply device for electric discharge machining. US 7148442B2. 
[8] Chen DS, Lin JK, Mai CC et al (2010) Green-energy power generator for electrical discharge machine. US 2010/0157637A1.

[9] Wu WJ (2002) Study and engineering design of the current mode electrical discharge machining power. Dissertation, Nanjing University of Aeronautics and Astronautics.

[10] Song BY, Wang YK, Zhao WS (2002) Study on circuit simulation of energy-saving pulse power of EDM. China Mech Eng 13:998-1000.

[11] Li CJ (2012) Research on energy saving pulse power supply and its floating threshold detection technologies for WEDM. Dissertation, Harbin Institute of Technology.

[12] Fan YS, Li CJ, Bai JC et al (2014) Experimental study on energy consumption of energy-saving pulse power for WEDM. Int J Adv Manuf Technol 72:1687-1691.

[13] Zhang LC, Wu J, Wang M et al (2015) A type of chopper type energy-saving pulse power of EDM. CN 204397103 U.

[14] Wu SP (2008) The research on the intelligent EDM pulse generator controller. Dissertation, China University of Petroleum.

[15] Wang KS, Gelgele HL, WangY et al (2003) A hybrid intelligent method for modeling the EDM process. Int J Mach Tools Manuf 43: 995-999.

[16] Kao CC, Shih AJ, Miller SF (2008) Fuzzy logic control of microhole electrical discharge machining. J Manuf Sci Eng 130: 064502-1-6.

[17] Wang WM, Zhao WS, Bai JC et al (1988) EDM discharge state detection and microcomputer adaptive control pulse power. Electromachining 2:34-41.

[18] Luo YF (2001) Research on intelligent control system of EDM process. Dissertation, Harbin Institute of Technology.

[19] Yan MT, Liao YS (1998) Adaptive control of the WEDM process using the fuzzy control strategy. J Manuf Syst 174: 263-274.

[20] Yan MT, Chen HT (2007) Monitoring and control of the micro wire-EDM process. Int J Mach Tools Manuf 47:148-157.

[21] Pei JY, Hu DJ, Gao CS et al (2001) Intelligent fuzzy control system applied in micro-EDM. Journal of Shanghai Jiaotong University 35: 1830-1833.

[22] Chen JM, Liu YH, Sang X (2009) Modeling and simulation of EDM intelligent control system. Comput Simul 26:181-184.

[23] Huang RN, Liu B, Lou YJ (2012) Research on micro EDM pulse generator with fuzzy control. China Mech Eng 23:1718-1722.

[24] Zhou M, Han FZ, Soichiro I (2008) A time-varied predictive model for EDM process. Int J Mach Tools Manuf 48:1668-1677.

[25] Yang Y, Yan D, Zhu XF et al (2013) Research of electric discharge machining approach based on intellectual control. Mach Tool Hydraul 41:75-78. 